\title{
Improved treatment of electron thermal conduction in plasma hydrodynamics calculations
}

\author{
Gregory A. Moses \\ Department of Nuclear Engineering, University of Wisconsin, Madison, Wisconsin 53706
}

\author{
James J. Duderstadt \\ Department of Nuclear Engineering, University of Michigan, Ann Arbor, Michigan 48104 \\ (Received 23 March 1976; final manuscript received 3 January 1977)
}

\begin{abstract}
The classical transport law for the hydrodynamic description of the electron heat flux breaks down in the nonequilibrium laser fusion plasma and an alternative expression for the electron heat flux vector must be used. The $a d$-hoc flux limit treatment used in present production level codes is analyzed and shown to provide physically plausible results but without providing any further insight into the properties of the plasma. Grad's 13 moment method is proposed as an alternative to the conventional Fourier's law expression for the heat flux. Numerical calculations confirm that this approach not only provides physically plausible results but provides them within a more fundamental framework than the ad-hoc flux limiting schemes and thus offers the possibility of gaining more insight into the flux limiting mechanism.
\end{abstract}

\section{INTRODUCTION}

Electron thermal conduction plays a very important role in the compression of fuel pellets to thermonuclear burn conditions using high powered lasers. ${ }^{1,2}$ To illustrate this, consider the typical laser fusion scenario for a simple spherical pellet of $\mathrm{D}-\mathrm{T}$ fuel irradiated uniformly on its surface with light from an intense laser source. The outer surface of the pellet will heat, ionize, and ablate outward forming a low density plasma cloud around the pellet core. The electrons in this cloud of plasma continue to absorb more laser radiation via inverse bremsstrahlung or plasma instability mechanisms. The laser radiation is only able to penetrate into the plasma atmosphere up to the "critical density" surface where the electron plasma frequency is equal to the incident laser frequency. The transport of energy from the critical density inward is by the mechanism of electron thermal conduction. As the pellet surface heats and ablates outward (blow-off) an ablation shock front is formed which converges (implodes) inward toward the center pushing cold D-T fuel ahead of it to higher and higher densities. Figure 1 distinguishes three regions in the plasma during the implosion process. Beyond the critical density the underdense plasma absorbs the laser energy and expands outward. Between the ablation front and the critical density, thermal conduction is the dominant energy transport mechanism which carries ene rgy from the absorption region into the ablation surface. Inside the ablation surface the D-T fuel is adiabatically compressed to Fermi degenerate electron densities. When the ablation front converges to the center, the super-dense pellet core is shock heated to initiate thermonuclear burn, and a thermonuclear burn wave propagates back outward through the dense core, self-sustained with recaptured energy from $3.5 \mathrm{MeV} \alpha$ particle reaction products. Thus, the laser is really used to compress the fuel pellet rather than to heat it. To obtain an optimum adiabatic compression, the laser pulse must be programmed in time such that the laser energy absorbed and transported in the pellet creates a thermal front that always remains just behind the ablation surface, thus driving it toward the pellet center without significantly heating the fuel ahead of it. If the laser energy is deposited too rapidly, the thermal front will move ahead of the ablation front, preheat the dense core, and degrade the compression. The key idea in laser fusion is to compress the fuel while keeping it relatively cold, only achieving significant temperatures once the pellet core has been compressed to very high densities. However, there is yet another constraint for if the thermal front lags too far behind the ablation surface, it will not drive the compression strongly enough to reach high densities, and the pellet atmosphere will thermally de-couple from the core and be heated to very high temperatures.

To demonstrate the strong dependence of optimum implosion on the value of thermal conductivity we use our one-dimensional code (see Appendix A), to simulate the implosion of a $400 \mu$ radius solid DT pellet with a shaped laser pulse. ${ }^{3}$ For the nominal value of thermal conductivity, $k_{e}$, we find a maximum compression of

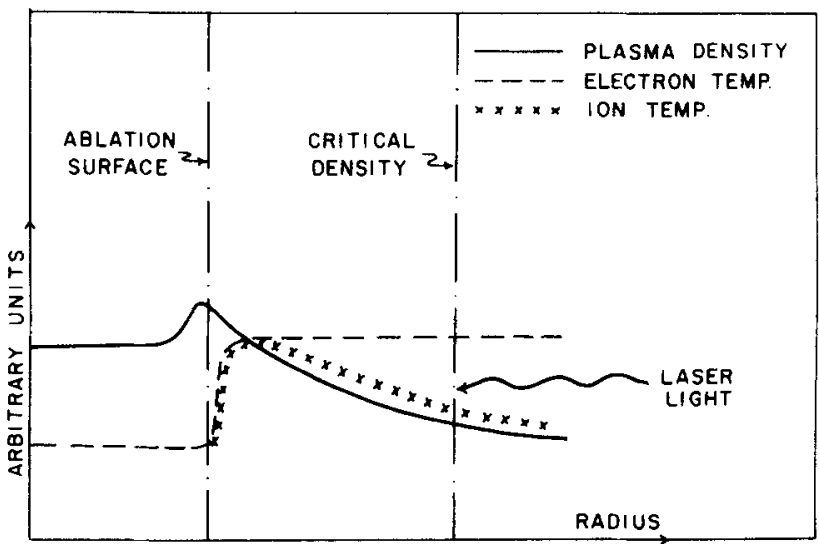

FIG. 1. Laser fusion pellet implosion by an intense laser source. Laser energy penetrates the plasma to the critical density surface, where it is carried to the ablation front by electron thermal conduction. Ahead of the ablation front the cold plasma is compressed to high densities. 
2800 times solid density. However, if we simply reduce $k_{e}$ by a factor of 10 then the compression for the same laser pulse is degraded to 100 times solid density. On the other hand, if $k_{e}$ is increased by a factor of 10 , the compression is reduced to less than 100 times solid density. Clearly, the laser profile can be adjusted to provide optimum compression for any value of $k_{e}$ however uncertainties in $k_{e}$ can substantially affect the final compression as we have demonstrated.

These considerations imply that a detailed understanding of the electron thermal conduction process is necessary to properly choose the parameters needed to obtain the optimum implosion, since it is this mechanism that transports energy from the underdense plasma corona region into the ablation surface, and therefore, is the key to laser driven pellet compression. The study of this mechanism cannot be decoupled from the remainder of the problem, however, because it is the close interaction of all of these nonlinear mechanisms that results in the laser-driven compression.

In Sec. II of this paper we shall review the mathematical models normally used to describe electron thermal conduction within a plasma hydrodynamics calculation. In Sec. III the shortcomings of this hydrodynamic model will be investigated and an alternative formulation will be suggested. Section IV will further describe this new model and present detailed numerical examples.

\section{A REVIEW OF THERMAL FLUX LIMITING SCHEMES IN PLASMA HYDRODYNAMICS}

For the purposes of this discussion we shall restrict our attention to the hydrodynamics used to describe the thermal components of the laser fusion plasma. In practice, these equations are usually augmented with separate treatments of nonthermal particles (suprathermal electrons and thermonuclear reaction products) and radiation and are solved numerically by large computer codes. ${ }^{4-6}$ We shall assume that the re are two thermal species, electrons and ions. The hydrodynamic equations describing the electrons and ions can be systematically obtained from the relevant kinetic equations (e.g., Boltzmann or Fokker-Planck) using the well known Chapman-Enskog scheme. That is, one can take moments of the Boltzmann equation with respect to collisional invariants, and expand the distribution function in a power series in Knudsen number (mean-free-path/ characteristic length) to complete the set of hydrodynamic equations with approximate transport laws. For example, the moment equation characterizing electron energy would take the form

$\frac{3}{2} n_{e} k_{B}\left(\frac{\partial}{\partial t}+u_{j} \frac{\partial}{\partial x_{j}}\right) T_{e}=-\frac{\partial}{\partial x_{i}} q_{e_{i}}-P_{e i j} \frac{\partial u_{j}}{\partial x_{i}}+Q_{c i}$

while the first order Chapman-Enskog expression for $q_{e i}$ is given by the familiar Fourier's law

$$
q_{e i}=-K_{e}\left(\partial T_{e} / \partial x_{i}\right) \text {, }
$$

where the electron thermal conductivity $K_{e}$ is usually taken as that calculated by Spitzer and Härm ${ }^{7}$

$$
K_{*}=\frac{20(2 / \pi)^{3 / 2}\left(k_{R} T_{e}\right)^{5 / 2} k_{B}}{m_{e}^{1 / 2} Z e^{4} \ln \Lambda} .
$$

It should be noted that the thermal conductivity is a strong function of temperature. It is also important to remember that this expression for the heat flux can only be considered valid for a plasma state near local thermodynamic equilibrium. Variations over space and time must be greater than local mean-free-paths and mean-free-times.

To illustrate some of the essential features of the thermal conduction process and the limitations of such a hydrodynamic description, consider the propagation of a temperature wave into an initially cold homogeneous and stationary plasma. The conventional hydrodynamic description of this process as represented by the nonlinear thermal conduction equation

$$
\frac{\partial T}{\partial t}=a \frac{\partial}{\partial x} T^{5 / 2} \frac{\partial T}{\partial x}+Q \delta(x) \delta(t),
$$

with

$$
a=\left(K_{0} / \frac{3}{2} n k_{B}\right), \quad K=K_{0} T^{5 / 2}, \int_{-\infty}^{\infty} T d x=Q
$$

predicts that a well-defined temperature front will propagate into the plasma ${ }^{8}$

$$
\begin{aligned}
T(x, t) & =\left(\frac{5}{18}\right)^{2 / 5}\left(\frac{Q^{2}}{a t}\right)^{2 / 9}\left(1-\xi^{2}\right)^{2 / 5}, & & \xi=\frac{x}{\left(a t Q^{5 / 2}\right)^{2 / 9}}<1, \\
& =0, & & \xi>1
\end{aligned}
$$

along the trajectory

$$
x_{f}=\left(a Q^{5 / 2} t\right)^{2 / 9}
$$

with a front propagation speed of

$$
\frac{d x_{f}}{d t}=\frac{2}{9}{\frac{\left(a Q^{5 / 2}\right)^{2 / 9}}{t^{7 / 9}}}^{2}
$$

The speed of the wave front slows as it propagates away from the source. Conversely, the front velocities approach infinity as $t-0$. This unphysical divergence of the front velocity is eliminated by noting that for Fourier's law to be valid, this solution must be considered only at times substantially greater than a mean collision time. This sets a lower time limit on the validity of this solution. For times less than this, we can expect the heat flux predicted by Fourier's law to be too large, since the front velocity is approaching infinity. A more physically realistic (order of magnitude) maximum value for the front velocity would be the thermal velocity of the electrons behind the front. In fact, there is a good correlation between all of these considerations displayed in Fig. 2. Here, the exact solution of the nonlinear diffusion equation is calculated. The time at which the front velocity is equal to the thermal velocity of the electrons behind the front is plotted along with the mean collision time of those same electrons. We see that the two times are different by only a factor of two and that the correlation is consistent for variations in $Q$ of six orders of magnitude. At times less than the collision time the nonlinear conduction equation predicts front velocities in excess of the electron thermal velocity while for times greater than the collision time, 


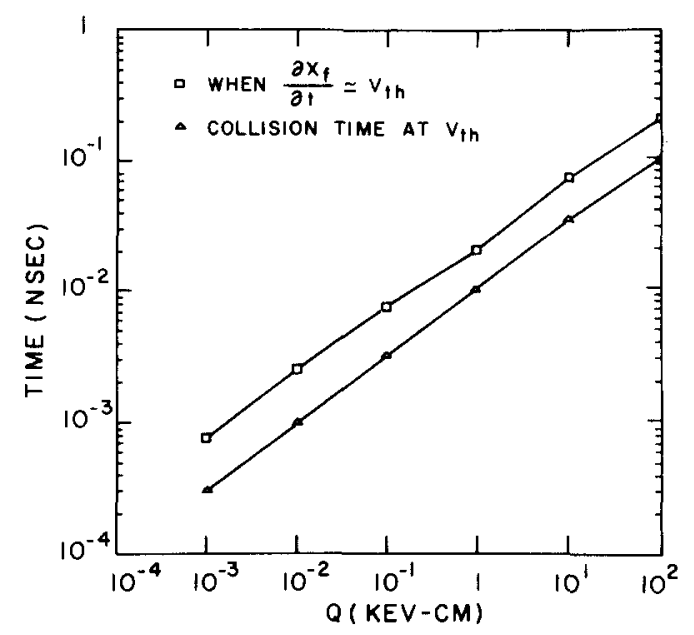

FIG. 2. Time when electron thermal speed equals thermal front speed $(a)$ and collision time of electrons at that thermal speed $(\Delta)$ for different source strengths.

the front velocity is less than the electron thermal velocity, which agrees with our physical intuition.

With regard to actual laser fusion calculations, we note that although the source is not a delta function in space or time, it can change on time scales comparable to the electron collision times, and hence the response of the plasma system will be analogous to this model problem. In particular, the mean time to collision for an electron in a $10^{21} \mathrm{~cm}^{-3}, 10 \mathrm{keV}$, Maxwellian plasma is approximately $10^{-10} \mathrm{sec}$. During the final 10-100 psec a laser pulse designed to provide optimum implosion will deliever up to $90 \%$ of its energy. The laser intensity and hence the electron energy source will increase orders of magnitude in times on the order of the electron mean time to collision. To describe the energy transport during this crucial time, account must be taken of the nonhydrodynamic nature of the relevant time and space scale lengths.

To correct the nonphysical predictions of the hydrodynamics equations as implemented in large scale computer codes when nonhydrodynamic conditions (large gradients or mean-free-path) are encountered, it has been customary for a number of years to impose an $a d-$ hoc limit on the thermal flux. It is usually argued that the thermal flux, $q$, can surely be no larger than the flow of electron kinetic energy across a surface in the absence of collisions. If one assumes that the electrons can be approximately characterized by an isotropic distribution, then this flux limit becomes ${ }^{9}$

$$
\mathrm{q}_{\max }=\left(\frac{1}{2} m_{e} \bar{v}^{2}\right) \quad\left(n_{e} \bar{v}_{x}\right)=\frac{3 \sqrt{3}}{8}\left(n_{e} k_{B} T_{e}\right)\left(\frac{k_{B} T_{e}}{m_{e}}\right)^{1 / 2} .
$$

Several alternative prescriptions are used, in practice, to implement such flux limiters. For example, a simple interpolation formula might be used ${ }^{4}$

$$
\mathrm{q}=-K \nabla T\left(1+\left|K \nabla T / \mathrm{q}_{\max }\right|\right)^{-1} .
$$

Another prescription commonly applied is simply to use

$$
\mathrm{q}=\min \left(-K \nabla T, \mathrm{q}_{\max }\right) .
$$

The significance of such ad-hoc limits on $q$ can be seen by futher investigation of our model heat conduction equation

$$
\rho c_{v} \frac{\partial T}{\partial t}=-\frac{\partial q}{\partial x} ; \quad \rho c_{v} \equiv \frac{3}{2} n_{e} k_{B} .
$$

Let

$$
\begin{aligned}
& q_{\max }=q_{0} T^{3 / 2} ; \quad q_{0}=\frac{3 \sqrt{3}}{8}\left(n_{e} k_{B}\right)\left(\frac{k_{B}}{m_{e}}\right)^{1 / 2} \\
& q=-\alpha K \nabla T-(1-\alpha) q_{\max } 0 \leq \alpha \leq 1 .
\end{aligned}
$$

This form for $q$ is essentially another way of interpolating between the diffusion and free streaming limits, depending on the value of $\alpha$. It is not suggested that this form of interpolation be used in calculations; however, it will greatly simplify the following analysis while preserving the essential character of the flux limiter. We need not give a specific expression for $\alpha(x)$ here for this will not be necessary for the purposes of the following discussion. Substituting in Eq. (10) we obtain

$\frac{\partial T}{\partial t}+\frac{3}{2}(1-\alpha) \frac{q_{0}}{\rho c_{v}} T^{1 / 2} \frac{\partial T}{\partial x}=\frac{\alpha}{\rho c_{v}} \frac{\partial}{\partial x} K \frac{\partial T}{\partial x}$.

If we define

$$
u_{L}=\frac{3}{2}(1-\alpha) \frac{q_{0}}{\rho c_{v}} T^{1 / 2},
$$

we see that as $\alpha \rightarrow 1$, we return to a parabolic diffusion equation

$$
\frac{\partial T}{\partial t}=\frac{K_{0}}{\rho c_{v}} \frac{\partial}{\partial x} T^{5 / 2} \frac{\partial T}{\partial x},
$$

where the front velocity can be approximated as

$$
v_{f} \simeq \frac{2 / 3 K_{0} T^{5 / 2}}{\rho c_{v}} \frac{1}{T} \frac{\partial T}{\partial x},
$$

while as $\alpha \rightarrow 0$ we are solving a hyperbolic advection equation

$$
\partial T / \partial t+u_{L}(\partial T / \partial x)=0,
$$

where the front velocity is $v_{f} \sim u_{L}$. The effect of averaging for $q$, Eq. (9), is to choose the smaller of these two velocities. Comparing $u_{L}$ with the thermal velocity of an electron at temperature $T$, we find

$$
\begin{aligned}
u_{L} & =\frac{3}{2}(1-\alpha) \frac{q_{0}}{\rho c_{v}} T^{1 / 2} \\
& =\frac{3}{2}(1-\alpha) \frac{1}{4}\left(\frac{3 k_{B}}{m_{e}}\right)^{1 / 2} T^{1 / 2}=\frac{3}{8}(1-\alpha) v_{\text {th }},
\end{aligned}
$$

where $v_{\text {th }}=\left(3 k_{B} T / m_{e}\right)^{1 / 2}$.

Hence, the pure advection equation $(\alpha=0)$ will propagate a wave at a velocity near the electron thermal velocity. The use of the conventional flux limiter prescription limits the velocity of the thermal front to approximately the thermal velocity of the electrons behind it. Thus, flux limiting insures that results fall within physically meaningful limits.

To study this modeled problem (i.e., no fluid motion) in more detail, we have numerically solved both the conventional and flux limited thermal conduction 


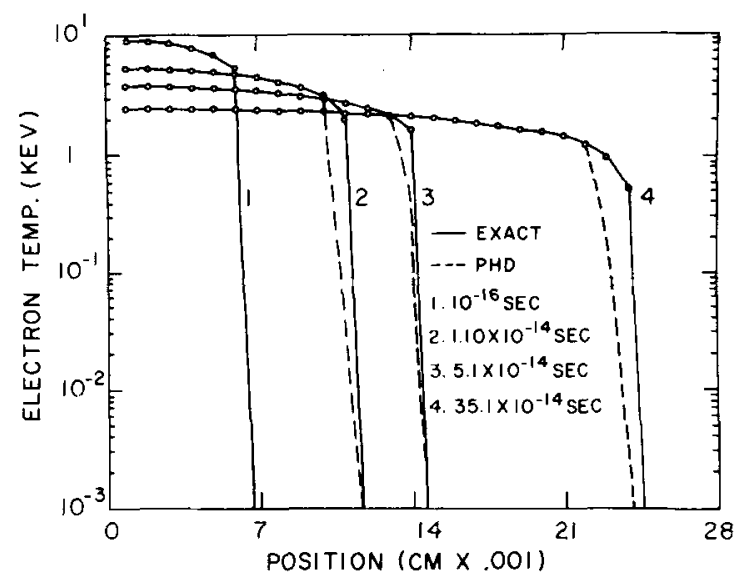

FIG. 3. Electron temperature profiles at different times calculated exactly and with the plasma hydrodynamics code.

problems using our plasma hydrodynamics code, (see Appendix). In Fig. 3, we first show the results of the conventional hydrodynamies treatment. The numerical solution could not be started at $t=0$ since for small times the temperature distribution is so narrow that to resolve it numerically would require a prohibitively small mesh. Instead, the exact solution at $t=10^{-15} \mathrm{sec}$ evaluated at the positions of the zone centers, is used as the initial temperature condition. In Fig. 3 we note that the temperature front has moved from about $6 \times 10^{-3}$ to $24 \times 10^{-3} \mathrm{~cm}$ in $3.509 \times 10^{-13} \mathrm{sec}$ giving it an average velocity of $5.1 \times 10^{10} \mathrm{~cm} / \mathrm{sec}$, which is greater than the speed of light! This is clearly an unphysical result; Fourier's law is predicting thermal conduction that is far too great. This is to be expected although because the mean free path and mean free time for a $8 \mathrm{keV}$ electron in this plasma are approximately $0.2 \mathrm{~cm}$ and 5.6 $\times 10^{-11} \mathrm{sec}$, respectively. Thus, we are trying to follow spatial and temporal behavior on a scale far too small to be treated properly with Fourier's law.

The effect of flux limited thermal conduction can be seen by repeating this calculation using the $a d-h o c$ flux limit given by Eq. (9). The results of this calculation are given in Fig. 4. In $36.1 \times 10^{-4} \mathrm{sec}$ the temperature profile has still not changed substantially from the initial profile. $1051.1 \times 10^{-14} \mathrm{sec}$ are required, as compared with $35.1 \times 10^{-14} \mathrm{sec}$ in the unlimited case, for the thermal front to move to $22 \times 10^{-3} \mathrm{~cm}$. This gives an average front speed of $1.5 \times 10^{9} \mathrm{~cm} / \mathrm{sec}$, while the thermal velocity of an $8 \mathrm{keV}$ electron is $3.7 \times 10^{9} \mathrm{~cm} / \mathrm{sec}$. Thus, the propagation speed is reduced to a value on the order of the electron thermal speed, as is expected. It is noted that the flux limit is not affecting the flux very much behind the thermal front; hence, the temperature profile is very flat for later times.

Care must be taken, however, when comparing these flux limited and nonflux limited examples as we have done. The plasma hydrodynamics code will produce the solution of the initial value problem

$$
\frac{\partial T}{\partial t}=a \frac{\partial}{\partial x} T^{5 / 2} \frac{\partial T}{\partial x}, \quad T(x, 0)=T_{0}(x),
$$

while the exact solution is provided for the equation

$$
\frac{\partial T}{\partial t}=a \frac{\partial T}{\partial x} T^{5 / 2} \frac{\partial T}{\partial x}+Q \delta(x) \delta(t)
$$

The fact that $T_{0}$ is a solution of Eq. (17) at $t=t_{0}$ provides the relation between these two problems. We may also consider Eq. (16) as the description of a physical problem where a certain amount of energy is concentrated in a plasma, providing a temperature profile $T_{0}$. Under proper physical conditions, Eq. (16) will describe the change of this temperature profile with time. These physical conditions must be such as to insure the validity of the thermal conduction model for the heat flux. If $T_{0}$ is a solution of Eq. (17) at $t=t_{0}$, we may test the validity of thermal conduction by comparing $t_{0}$ with the collision time of the electrons behind the front. If $t_{0}$ is greater than the collision time, then we found that thermal conduction probably adequately describes the heat flux. From Fig. 2 it is seen that an equivalent test is a comparison of front velocity to thermal electron velocity, for $v_{f}<v_{t h}$, thermal conduction is valid. If $T_{0}$ is not a solution of Eq. (17) at $t=t_{0}$, we cannot make the comparison $t_{0}>t_{m}$ where $t_{m}$ is the mean-free-time, since $t_{0}$ is only defined with regard to the solution of $\mathbf{E q}$. (17). In this case, other considerations for thermal conduction validity must be used, namely, $v_{f}<v_{\text {th }}$. That the hydrodynamics code with flux limiting is accurately describing the time evolution from $T(x, 0)$ must be deduced from other, as yet unspecified considerations. Thus, the only hope for describing heat flux when Fourier's law is not valid must come from more basic theoretical principles.

\section{AN ALTERNATIVE TREATMENT OF ELECTRON THERMAL CONDUCTION}

The $a d-h o c$ nature of the conventional prescription for thermal flux limiting motivates us to search for a more fundamental scheme for modifying the conventional hydrodynamics equations to account for nonhydrodynamic phenomena. The particular problem we will attempt to describe involves the rapid heating of the corona electrons (near the critical density) in a fuel pellet by a laser pulse which may rise several orders of magnitude in intensity in times of $10-100$ psec. Heat will then be transported into the pellet core by strong ther-

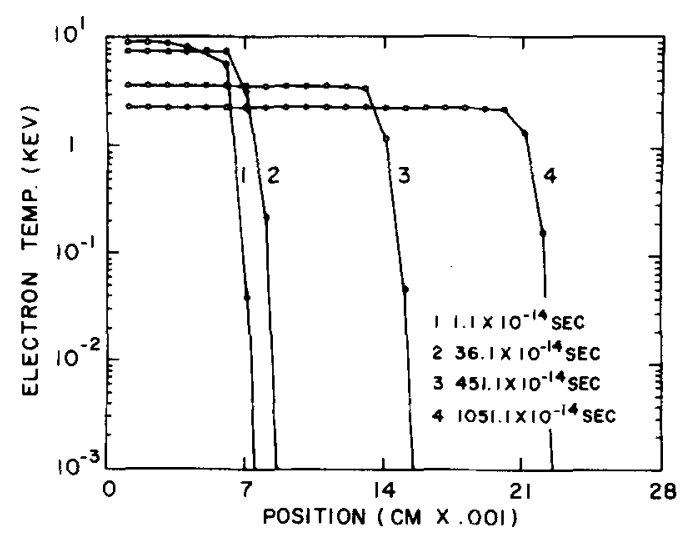

FIG. 4. Electron temperature profiles at different times calculated from the flux limited temperature diffusion equation by the plasma hydrodynamics code. 
mal conduction characterized by a sharp wave front. We have noted that the mean free time for a $10 \mathrm{keV}$ electron at $10^{21} \mathrm{~cm}^{-3}$ density is approximately $100 \mathrm{psec}$; hence, the electron heating can occur on time scales comparable in magnitude to the electron collision times, indicating the possibility of nonequilibrium electron distribution. Clearly, the conventional hydrodynamic treatment (Fourier's law) of energy transport will be invalid on such short time scales.

To understand this, recall that the Chapman-Enskog technique often employed to obtain approximate transport laws for $P_{i j}$ and $q_{i}$ assumes that the distribution function can be formally expanded in an infinite series of distribution functions [of increasing order in (mfp/ characteristic length)]

$$
f(\mathbf{x}, \mathbf{v}, t)=\sum_{n=0}^{\infty} \epsilon^{n} f^{(n)}(\mathbf{x}, \mathbf{v}, t)
$$

where it is assumed that

$$
f^{(0)}(\mathbf{x}, \mathbf{v}, t)=n(\mathbf{x}, t)\left(\frac{m}{2 \pi k_{B} T}\right)^{3 / 2} \exp \left(-\frac{m|\mathbf{v}-\mathbf{u}|^{2}}{2 k_{B} T}\right)
$$

is the local Maxwellian or equilibrium distribution. Usually, the expansion is truncated after two terms resulting in the so-called Navier-Stokes level of description. A key assumption, however, is that the distribution function is a slowly varying function of time, where "slowly" implies variation on macroscopic time scales much longer than collision times such that

$$
f(\mathbf{x}, \mathbf{v}, t)=f[\mathbf{x}, \mathbf{v} \mid n(\mathbf{x}, t), \mathbf{u}(\mathbf{x}, t), T(\mathbf{x}, t)] .
$$

This assumption limits this successive approximation solution of the Boltzmann equation, and hence the expressions for $P_{i j}$ and $q_{i}$, to slow time variation of the distribution function. We noted earlier that the electrons gain energy very quickly and the energy transport is characterized by steep temperature gradients, also indicating the presence of rapid time variation of the distribution function. Under these circumstances, the Chapman-Enskog method does not seem appropriate to estimate the distribution function. In fact, to any order in the infinite series, $f^{(n)}$, this same slow time variation assumption is made, so higher order ChapmanEnskog terms will not help to obtain better estimates. The solution for the distribution function must instead be obtained without the slow time variation assumption.

An alternative technique for formally solving the Boltzmann equation is Grad's moment method. ${ }^{10}$ This method is not necessarily restricted to slow time variations in the distribution function and the refore seems better suited for the treatment of rapidly varying thermal sources. In Grad's method, the distribution function (written for a single species for convenience) is expanded in velocity moments about a local Maxwellian distribution

$$
f(\mathrm{x}, \mathrm{v}, t)=f^{(0)} \sum_{n=0}^{\infty} \frac{1}{n !} a_{[i]}^{(n)}(\mathrm{x}, t) H_{[i]}^{(n)}(\mathrm{v}) .
$$

$H_{[i]}^{(n)}$ is a Hermite polynomial of $n$th degree and $H_{[i]}^{(n)}$ and $a_{[i]}^{(n)}$ are tensors of $n$th rank. ${ }^{11}$ Due to the orthogonality properties of Hermite polynomials, the inversion formula for the coefficients is

$$
a_{[i]}^{(n)}=1 / n(\mathbf{x}, t) \int f(\mathbf{x}, \mathbf{v}, t) H_{[i]}^{(n)} d^{3} v
$$

The first few $a_{[i]}^{(i)}$ can be related to the familar definitions for density, fluid velocity, temperature, pressure tensor, and heat flux.

When the expansion, Eq. (18), is substituted into the Boltzmann equation and integrated over $d^{3} v$, an infinite set of first order partial differential equations for the $a_{[i]}^{(n)}$ is obtained. If the equations up to $a_{i j k}^{(3)}$ are retained, there are a total of twenty coupled equations. In fact, the equations never decouple for any order $\alpha^{(n)}$ because in general, $a_{[i]}^{(n+1)}$ will appear in the equation for $\partial a_{[i]}^{(n)} / \partial t$. To close the set, the $a_{[i]}$ term is evaluated formally using an ansatz for the distribution function that is complete up to the $n$th term in the infinite series expansion.

This system of equations is typically reduced to thirteen by contracting the third rank tensor $\left(a_{i j k}^{(3)} \rightarrow a_{i r r} \rightarrow q_{i}\right)$ to a vector that can be related to the thermal heat flux. The thirteen coefficients (i.e., variables in the moment equations) are then $n, u_{1}, u_{2}, u_{3}, P_{11}, P_{22}, P_{33}, P_{12}=P_{21}$, $P_{23},=P_{32}, P_{31}=P_{13}, q_{1}, q_{2}$, and $q_{3}$, while the corresponding ansatz for the distribution function takes the form

$$
f(\mathrm{x}, \mathrm{v}, t)=f^{(0)}\left[1+p_{i} \frac{U_{i} U_{j}}{p \bar{v}^{2}}+4 q_{i} U_{i} \frac{\left(U_{j} U_{j}-5 / 2 \bar{v}^{2}\right)}{5 P \bar{v}^{4}}\right]
$$

where $P_{i j}=p_{i j}+\delta_{i j} P ; \quad U_{i}=v_{i}-u_{i} ; \quad P=\frac{1}{3} P_{r r} ; \quad \bar{v}=\left(3 k_{B} T\right)$ $m)^{1 / 2}$. The resulting set of thirteen moment equations is then comprised of the conventional hydrodynamic equations of mass, momentum, and energy conservation

$$
\begin{aligned}
& \frac{\partial \rho}{\partial t}+u_{i} \frac{\partial \rho}{\partial x_{i}}+\rho \frac{\partial u_{i}}{\partial x_{i}}=0, \\
& \rho\left(\frac{\partial}{\partial t} u_{i}+u_{j} \frac{\partial u_{i}}{\partial x_{j}}\right)=-\frac{\partial P_{i}}{\partial x_{j}}, \\
& \frac{3}{2} n k_{B}\left(\frac{\partial T}{\partial t}+u_{i} \frac{\partial T}{\partial x_{i}}\right)=-\frac{\partial q_{i}}{\partial x_{i}}-P_{i j} \frac{\partial u_{j}}{\partial x_{i}},
\end{aligned}
$$

augmented by two additional equations which represent generalizations of the traditional transport laws

$$
\begin{aligned}
& \frac{\partial P_{j k}}{\partial t}+u_{i} \frac{\partial P_{j k}}{\partial x_{i}}+P_{i k} \frac{\partial u_{j}}{\partial x_{i}}+P_{i j} \frac{\partial u_{k}}{\partial x_{i}}+P_{j k} \frac{\partial u_{i}}{\partial x_{i}}+\frac{\partial P_{i j k}}{\partial x_{i}} \\
& =R_{P_{j k}}, \\
& \frac{\partial q_{k}}{\partial t}+u_{i} \frac{\partial q_{k}}{\partial x_{i}}+\frac{7}{5} q_{i} \frac{\partial u_{k}}{\partial x_{i}}+\frac{7}{5} q_{k} \frac{\partial u_{i}}{\partial x_{i}}+\frac{2}{5} q_{j} \frac{\partial u_{j}}{\partial x_{k}} \\
& \quad+P \frac{\partial\left(p_{j k} / \rho\right)}{\partial x_{i}}+\frac{5}{2} \frac{p_{i k}}{\rho} \frac{\partial k_{B} T}{\partial x_{i}}+\frac{5}{2} \frac{P}{m} \frac{\partial k_{B} T}{\partial x_{k}} \\
& \quad-\left(p_{j k} / \rho\right) \frac{\partial p_{i k}}{\partial x_{i}}=R_{\alpha_{k}},
\end{aligned}
$$

where

$R_{a_{k}}=\int d^{3} v(m / 2) U_{j} U_{j} U_{k} J(f, f), \quad R_{p_{f k}}=\int d^{3} v m U_{j} U_{k} J(f, f)$,

while $J(f, f)$ is the collision term (e.g., Boltzmann or Fokker-Planck).

Since our concern is with thermal conduction process- 
es, we will focus our attention on Eq. (25). We suggest that the unphysical infinite heat flux predicted by Fourier's law can be corrected by including higher order conservation equations, as given by Grad's method, in the description of the dynamic behavior of the plasma. This constitutes a systematic application of a classical technique for solving the Boltzmann equation. Since the conventional hydrodynamic equations are, in fact, the result of the same technique, we are only suggesting that within the same framework, we carry the application to the next higher order equations. We note that this is not the same as using higher order ChapmanEnskog terms because we are particularly concerned with the time dependence. Since energy is added to the system on time scales comparable to the electron mean free time, we suspect that the functional dependence $f\left(\mathbf{x}, \mathbf{v} \mid n, u_{i}, T, p_{i j}, q_{i}\right)$ is a better representation of the distribution function than is $f\left(x, v \mid n, u_{i}, T\right)$.

The equation governing the time dependent heat flux as it is written in Eq. (25) is unsuitable for further detailed analysis, therefore some approximations and additions must be made. To model the plasma we chose the one-fluid two-temperature description, hence the single fluid velocity will be that of the more massive ions. Within this context, all convective terms in the electron heat flux equation will be regarded as small in this analysis. This can be justified by noting that the electron conduction characteristic velocity, $v_{e}$, is the electron thermal velocity whereas the convection characteristic velocity is the ion thermal velocity, $v_{i}$. Thus,

$$
v_{i} / v_{\bullet} \sim\left(m_{\mathbf{e}} / m_{i}\right)^{1 / 2} \sim 1 / 60
$$

for a deuterium plasma. We also recognize that the electron viscosity is generally much smaller than the ion viscosity so since we are treating the electrons in this analysis, we shall also assume that viscosity terms are small. We anticipate that the dominant feature in our analysis will be the time dependent heat flux. These assumptions greatly simplify the heat flux equation

$$
\frac{\partial q_{k}}{\partial t}+\frac{5}{2} \frac{P}{m} \frac{\partial k_{B} T}{\partial x_{k}}=R_{\alpha_{k}} .
$$

To render this equation suitable for a general numerical treatment, we use a simple $\mathrm{BGK}$-like approximation for the collision integral

$$
R_{\tau_{k}}=-q_{k} / \tau,
$$

where $\tau$ is some characteristic relaxation time (such as the electron-ion mean collision time)

$$
\tau=3 \sqrt{m_{e}} T_{e}^{3 / 2}\left(4 \sqrt{2 \pi} n_{e} \ln \Lambda e^{4}\right)^{-1} .
$$

The simplified flux equation is then

$$
\frac{\partial q_{k}}{\partial t}+\frac{5}{2} \frac{P k_{B}}{m} \frac{\partial T}{\partial x_{k}}+\frac{q_{k}}{\tau}=0 .
$$

Several remarks can be made about Eq. (27).

(i) In the absence of macroscopic spatial gradients, the heat flux decays from an initial condition to zero with a decay rate of $1 / \tau$. This is plausible because collisions will isotropize the distribution function and relax it to a local Maxwellian on this time scale. This also indicates a significant difference between the equations for density, fluid velocity, and temperature and the equations for the pressure tensor and heat flux. Since the first three equations are the result of moments using collisional invariants, no collision term explicitly appears in them. For this reason, their time response is governed by macroscopic gradients and time scales. The heat flux equation contains a collision term, however, so the time dependent heat flux is described by this equation on time scales comparable to an electron mean free time. Hence, by including the time dependent heat flux in the functional dependence of the distribution we include the short time scale response of the distribution function.

(ii) For steady state, the heat flux equation reduces to

$$
q_{k}(\mathrm{x}, t)=-\frac{5}{2} \frac{P k_{\mathrm{B}} \tau}{m} \frac{\partial T}{\partial x_{k}}
$$

which is in the form of Fourier's law; hence, a thermal conductivity can be defined as

$$
K=\frac{5}{2} P k_{B} \tau / m \text {. }
$$

In fact, similar results for thermal conductivity are obtained using the Chapman-Enskog technique and Grad's method, but using an elaborate evaluation of the collision term.

Our contention is that the thirteen moment equations can be used, in their fully time dependent form, to better describe the time evolution of the plasma through their additional state variables. The problem of evaluating the thermal conductivity still remains in this analysis and more elaborate methods than the simple BGK model have been used (by Salat for instance) $)^{12}$ to approximate the thermal conductivity.

There are several similarities between the proposed treatment (including the heat flux equation along with the temperature equation) and the conventional flux limited temperature diffusion. Recall that the flux limited heat flux is given by

$$
\mathbf{q}=-K \nabla T\left(1+\left|K \nabla T / \mathbf{q}_{\max }\right|\right)^{-1},
$$

where $q_{\max }$ is independently estimated. If for a moment we approximate the time scale as

$$
\gamma \equiv q(\partial q / \partial t)^{-1},
$$

where $\gamma$ is the characteristic time over which the flux is changing, then Eq. (27) reduces to a form very similar to that of Eq. (28)

$$
\mathrm{q}=-K \nabla T /(1+|\tau / \gamma|)
$$

except that in our thirteen moment description the flux is limited by the ratio of two time scales while in Eq. (28) it is limited by the ratio of two fluxes. Of course, $\gamma$ is really a function of the fluxes indicated by Eq. (29). In conventional flux limiting, the reduction in the flux is dependent upon the choice of $\mathrm{q}_{\max }$. In the thirteen moment method all terms are at least formally defined within the framewrok of the thirteen-moment approximation. For simplicity, in our treatment estimates of 


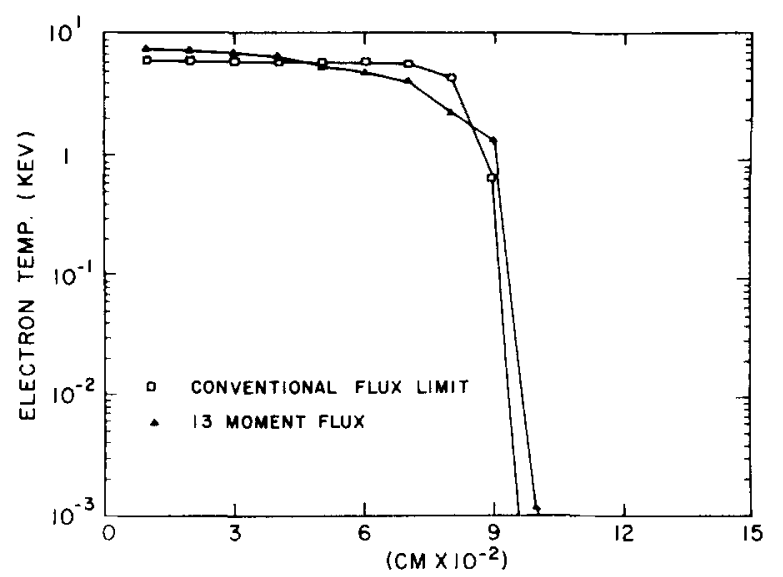

FIG. 5. Electron temperature calculated from the flux limited temperature diffusion equation (a) and the standard temperature diffusion equation augmented by the thirteen-moment heat flux equation $(\Delta)$ for a strongly flux limited problem.

such terms as thermal conductivity are not necessarily calculated in full consistency with the thirteen-moment formalism but, in principle, they could be.

Going back to the original equation, Eq. (27), we can write

$$
q_{k}=-\frac{5}{2} \frac{P k_{B}}{m}\left(\frac{1}{1 / \tau+1 / \gamma}\right) \frac{\partial T}{\partial x_{k}} .
$$

This shows explicitly that the effect of the thirteen-moment treatment is to select the smaller of the two time scales, $\tau$ and $\gamma$. The situation where $\gamma<\tau$ will result in less flux dependence on spatial temperature gradients than predicted by considering only the collision time. This situation will only occur if the time behavior of the flux is driven by some external source with $\gamma$-like time behavior. This is indeed the case in laser fusion plasmas as has been demonstrated in Sec. II. In a qualitative sense, the conventional flux limit is treating the symptom of the problem, while this method is treating the cause. We suspect then, that the thirteen moment method offers the opportunity to gain greater understanding of the flux limit mechanism.

\section{SOME NUMERICAL RESULTS}

We must now consider the problem of solving the coupled set of nonlinear partial differential equations

$$
\begin{aligned}
& \frac{3}{2} n k_{B} \frac{\partial T}{\partial t}=-\frac{\partial}{\partial x} q+S, \\
& \frac{\partial q}{\partial t}+\frac{5}{2} \frac{P k_{B}}{m} \frac{\partial T}{\partial x}+\frac{q}{\tau}=0 . \\
& \tau\left(T^{3 / 2}\right), P(T) .
\end{aligned}
$$

We do this by solving Eq. (33) using the finite difference technique and an initial guess for $T$. From the solution for $q$, we can calculate

$$
\gamma=q(\partial q / \partial t)^{-1} \text {. }
$$

We write Eq. (32) as

$$
\frac{3}{2} n k_{B} \frac{\partial T}{\partial t}=\frac{\partial}{\partial x} \frac{K}{1+\tau / \gamma} \frac{\partial T}{\partial x}+S
$$

and using the estimate for $\gamma(x, t)$ from the solution of Eq. (33) we solve this nonlinear diffusion equation. With this calculated value of $T(\mathrm{x}, t)$, we can test for convergence with the heat flux equation by demanding

$$
\frac{K\left(T^{5 / 2}\right)}{1+\tau\left(T^{3 / 2}\right) / \gamma} \simeq q(x, t)
$$

where the left-hand side is evaluated from the temperature equation and the right-hand side from the heat flux equation. This is done point-wise in the finite difference mesh.

A calculation similar to those described in Sec. II was used to compare the conventional flux limit with the thirteen-moment treatment. The initial temperature distribution was allowed to relax for $10^{-11} \mathrm{sec}$ as shown in Figs. 5 and 6 . For a $5 \mathrm{keV}$ electron in a $10^{21} \mathrm{~cm}^{-3}$ plasma, the mean-free-path and mean-free-time are $360 \mu \mathrm{m}$ and $12 \mathrm{psec}$, respectively. We see that this problem is very far from the range of hydrodynamic validity in space and time. Changes are followed over distances that are very short compared with the meanfree-path and over times less than the mean-free-time. It is seen that the thirteen-moment treatment gives results that are nearly the same as the conventional flux limit, whereas the normal hydrodynamic description with Spitzer thermal conductivity would have given nonsensical results. The thirteen-moment temperature profile is more rounded because the heat flux is limited by the time variation constraint at all mesh points along the profiles, whereas the conventional flux limit only affects the flux near the steep spatial gradient, the electrons behind the heat front, where gradients are not steep, become isothermal faster than a collision time. So, although the conventional flux limit treats the propagation of the temperature front in a plausible manner, it misses the details of temperature relaxation behind the front.

Most important is the fact that the thirteen-moment formalism, along with a very simple treatment of the collision integral, yields physically reasonable results in a situation where the conventional Navier-Stokes equations break down. The good agreement between the

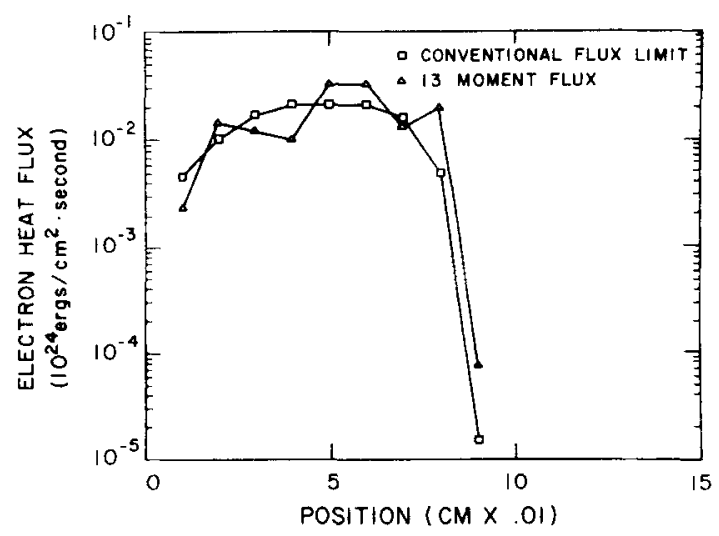

FIG. 6. Electron heat flux calculated from the flux limited temperature diffusion equation $(a)$ and the temperature diffusion equation augmented by the thirteen-moment heat flux equation $(\Delta)$ for a strongly flux limited problem. 
thirteen-moment treatment and the conventional flux limit emphasizes the utility of the flux limit as a computational device; however, such ad-hoc flux limiters must still be considered an artifical device by which physically reasonable answers are extracted from nonhydrodynamic conditions.

Spatially, the thirteen-moment method is expected to be valid only for the mean-free-path less than the characteristic scale length (in the same sense as the validity of the conventional hydrodynamic equations); however, this example indicates that it does well for scales much smaller than the mean-free-path. This again indicates that the virtue of using a particular asymptotic approximate solution of the Boltzmann equation is finally determined by trying it and evaluating the results.

The saw-toothed nature of the flux using the thirteenmoment method is characteristic of the numerical solution of convective type equations. With only first derivatives in space, the finite difference formula will propagate this saw-tooth form and without dissipation it will not decay. Since our numerical algorithm is solving two non-linear coupled convective-like equations, this behavior is not surprising.

In a more sophisticated application we applied the thirteen-moment treatment of electron thermal conduction implemented within the hydrodynamics code to analyze the slab target experiment to show the anomalous reduction of thermal conductivity, ${ }^{13}$ At this point, rather than trying to reproduce or add to the explanation of the experimental results, we were only interested in comparing the thirteen-moment treatment with the conventional flux limited treatment. We found that the thirteen-moment and flux limited calculations were identical for normal scaling of the flux limit, but they were just at the point where a reduction of the flux limit would affect the energy transport. We then reduced the flux limit by a factor of twenty and also reduced the only adjustable parameter in the thirteen-moment treatment, $\tau$, by a factor of 20 . The temperature profiles at 560 psec are shown in Fig. 7. We see that they are nearly

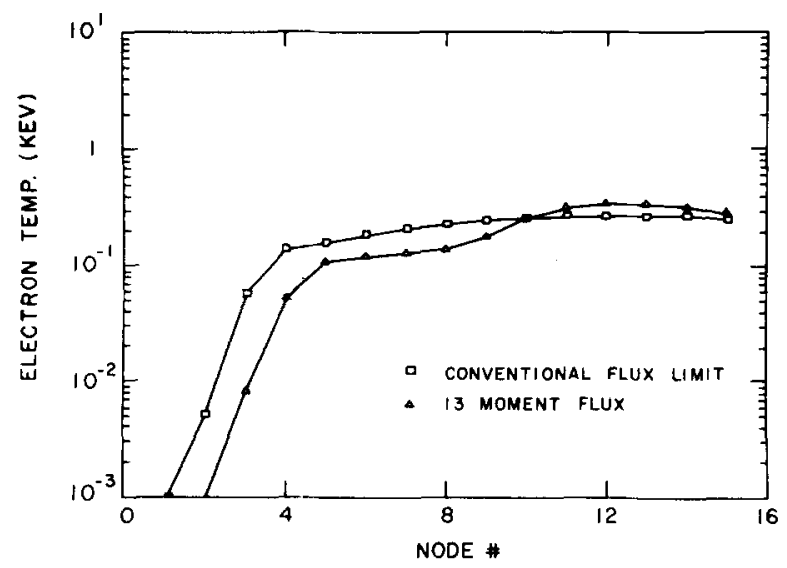

FIG. 7. Electron temperature for a $15 \mathrm{~J}$ Gaussian laser pulse incident on a slab of $\mathrm{CH}_{2}$, calculated from the flux limited temperature diffusion equation ( $(0)$ and the temperature diffusion equation augmented by the thirteen-moment heat flux equation $(\Delta)$.

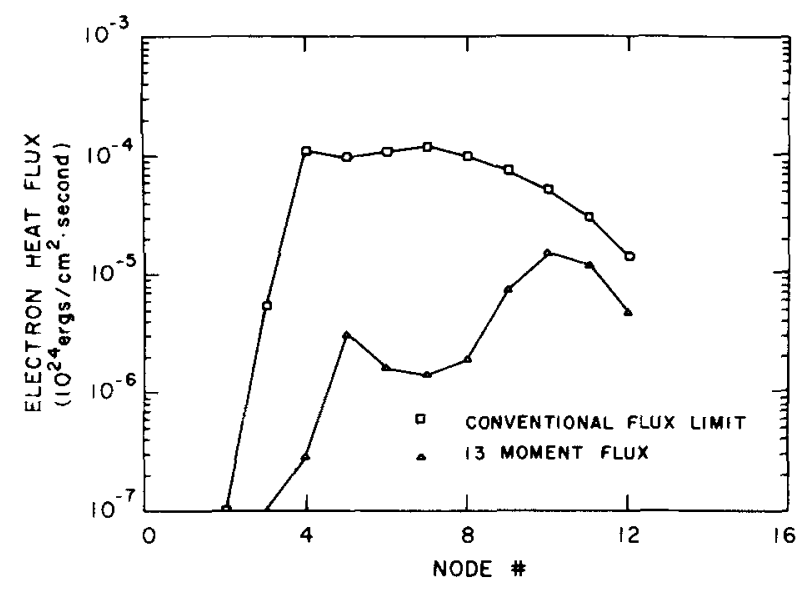

FIG. 8. Electron heat flux profiles calculated from the flux limited temperature diffusion equation (a) and the temperature diffusion equation augmented by the thirteen-moment heat flux equation $(\Delta)$.

the same, but the plots of the corresponding heat flux in Fig. 8 indicate very different results for the two types of calculation. The conventional flux limit treatment produces flux values behind the front that are at least an order of magnitude higher indicating a much greater skewness of the distribution function than is predicted by the thirteen-moment method. At no time in the thirteen-moment calculation is the flux limited by $\gamma$, but rather it is only reduced by the effective reduction of the thermal conductivity due to its dependence on $\tau$. This was not the case in the earlier example where the flux was most definitely restricted by the ratio of $\tau / \gamma$ rather than the absolute value of $\tau$. This is consistent with the observation that the thermal flux in the target is anomalously reduced below a value that was hydrodynamically plausible.

\section{CONCLUSIONS}

By investigating the breakdown of classical hydrodynamics in describing electron energy transport away from a strong heat source (e.g., in a laser heated plasma) we conclude that at least part of the problem rests in the treatment of the time dependence of the distribution function. Classical Chapman-Enskog methods for transport law calculations do not adequately treat this rapid time dependence. However, the Grad thirteen moment equations do provide rapid time response through a time dependent diffe rential equation for the heat flux in addition to the standard hydrodynamic equations. A simple numerical example confirms that the thirteen-moment method provides physically plausible results where the Navier-Stokes description does not and suggests that the thirteen moment equations might play a significant role in breaching the gap between hydrodynamics and kinetic theory in this special problem of strong electron thermal conduction. In addition, this method represents a practical approach to the flux-limited calculations because it can be implemented in a full scale laser-plasma hydrodynamics code, as we have demonstrated with our numerical examples. 


\section{ACKNOWLEDGMENTS}

Work supported in part by the National Science Foundation.

\section{APPENDIX}

The plasma hydrodynamics code solves the one fluid, two temperature equations of hydrodynamics in one dimensional (slab, cylindrical, and spherical) geometry, and in Lagrangian coordinates. The electrons and ions are assumed to move with a single fluid velocity implying no charge separation. The electrons and ions may each have their own Maxwellian distribution about dif ferent temperatures however, necessitated by the slow temperature relaxation time of the electrons and ions as compared with self-relaxation. The momentum equation is solved using an explicit finite difference technique with the addition of artificial viscosity. The energy equations include flux limited thermal diffusion through the electrons and ions, work on the electrons and ions, collisional coupling between the electrons and ions, and source terms. The electron source term is due to laser light absorption which is calculated using an inverse bremsstrahlung attenuation up to the critical density and there, a specified fraction of the remaining energy can be deposited before the attenuation of the re flected energy is calculated. Transport and absorption coefficients used in these equations are the standard Maxwell averaged quantities for thermal conduction, electron-ion relaxation, and inverse bremsstrahlung absorption. The nonlinear temperature equations for electrons and ions are solved simultaneously using implicit finite difference techniques with iteration allowed for re-evaluation of the nonlinear coefficients in the equations. Ideal gas equations of state are used to close the set of equations.

${ }^{1} \mathrm{~J}$. Nuckolls, L. Wood, A. Thiessen, and G. Zimmerman, Nature (London) 239, 139 (1972).

${ }^{2}$ K. Brueckner and S. Jorna, Rev. Mod. Phys. 46, 325 (1974)

${ }^{3}$ J. Lindl, Nucl. Fusion 14, 511 (1974).

${ }^{4} \mathrm{G}$. Zimmerman, Comments Plasma Phys. Controlled Fusion 2,85 (1975).

${ }^{5}$ G. Fraley, E. Linnebur, R. Mason, and R. Morse, Phys. Fluids 17, 474 (1974).

${ }^{6}$ E. Goldman, Plasma Phys. 15, 289 (1973).

${ }^{7}$ L. Spitzer, Physics of Fully Ionized Gases (Wiley, New York, 1962), 2nd ed., p. 144.

${ }^{8} \mathrm{Y}$. Zel'dovich and Y. Raizer, Physics of Shock Waves and High Temperature Hydrodynamic Phenomena (Academic, New York, 1966), Vol. II, p. 664.

${ }^{9}$ R. Morse and C. Nielson, Phys. Fluids 16, 830 (1970).

${ }^{10} \mathrm{H}$. Grad, in Handbuch der Phvsik, edited by $\mathrm{S}$. Flügge (Springer Verlag, Berlin, 1958), Vol. 12, p. 205.

${ }^{11}$ H. Grad, Commun. Pure Appl. Math. 2, 325 (1949).

${ }^{12}$ A. Salat, Plasma Phys. 17, 589 (1975).

${ }^{13}$ R. Malone, R. McCrory, and R. Morse, Phys, Rev. Lett. 34, $721(1975)$ 